\title{
Methods for 3D digitization of Cultural Heritage
}

\author{
George Pavlidis ${ }^{\mathrm{a}, *}$, Anestis Koutsoudis ${ }^{\mathrm{a}}$, Fotis Arnaoutoglou ${ }^{\mathrm{a}}$, Vassilios Tsioukas ${ }^{\mathrm{b}}$, \\ Christodoulos Chamzas ${ }^{\mathrm{c}}$ \\ ${ }^{a}$ Cultural and Educational Technology Institute, Research Center ATHENA, 58 Tsimiski Street, 67100 Xanthi, Greece \\ ${ }^{\mathrm{b}}$ Department of Architectural Engineering, Democritus University of Thrace, 12 Vas. Sofias Street, 67100 Xanthi, Greece \\ ${ }^{c}$ Department of Electrical and Computer Engineering, Democritus University of Thrace, 12 Vas. Sofias Street, 67100 Xanthi, Greece
}

Received 22 May 2006; accepted 31 October 2006

\begin{abstract}
Complete digital recording of Cultural Heritage is a multidimensional process. It depends highly on the nature of the subject of recording as well as the purpose of its recording. The whole process involves the three-dimensional digitization, digital data processing and storage, archival and management, representation and reproduction. In this paper we briefly review methods for three-dimensional digitization that are applicable to cultural heritage recording.
\end{abstract}

(C) 2007 Elsevier Masson SAS. All rights reserved.

Keywords: 3D digitization; Cultural heritage recording

\section{Introduction}

Complete recording of Cultural Heritage is a multidimensional process. It addresses not only the problem of threedimensional (3D) digitization of objects and monuments but involves all the aspects of this new digital content management, representation and reproduction. It addresses issues affecting the whole life cycle of the digital cultural content. Five main processes can be identified in digital recording. These processes are shown graphically in Fig. 1. All there processes have their own demands for advanced algorithms, new hardware and more sophisticated software implementations.

3D digitization of cultural heritage is the first step of the overall process of the complete recording of objects and monuments. It consists of multiple processes and exhibits variations in accordance with specific application requirements. Due to the complexity of the digitization needs that emerge

\footnotetext{
* Corresponding author. Tel.: +30 2541 078787x225; fax: +30 2541063656.

E-mail addresses: gpavlid@ceti.gr (G. Pavlidis), akoutsou@ceti.gr (A. Koutsoudis), fotarny@ceti.gr (F. Arnaoutoglou), vtsiouka@arch.duth.gr (V. Tsioukas), chamzas@ee.duth.gr (C. Chamzas).
}

from the objects themselves, there is a plethora of methods and technologies. The target of every such technique is to address successfully a particular type of objects or class of objects or monuments, or to fulfill particular demands and needs of a specific digital recording project (i.e. complete recording for archiving, digitization for presentation, digitization for commercial exploitation).

The plethora of available 3D digitization systems is the result of three main factors that influence the suitability and the applicability of a method:

\section{Complexity in size and shape}

2. Morphological complexity (level of detail)

3. Diversity of raw materials

There are techniques with satisfying results for microscopic objects, others for small, medium and large objects and others for monuments. There are different techniques for ceramic or metallic or glass objects. It should also be noted that techniques with satisfying results for one kind of objects. An extensive study on the available methods for 3D digitization of the cultural heritage has been done in our Institute. One 


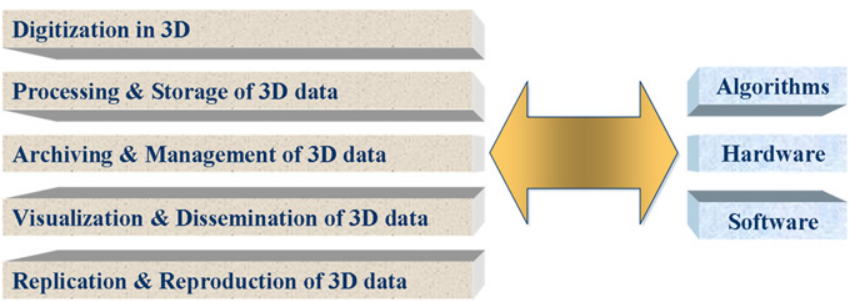

Fig. 1. Complete recording of cultural heritage.

of the important outcomes of this study is the construction of the Nine-Criteria Table (Table 1), which summarizes the possible parameters for choosing a 3D digitization system for cultural heritage applications.

3D digitization is a complex process that consists mainly of three phases:

1. Preparation, during which certain preliminary activities take place that involve the decision about the technique and methodology to be adopted as well as the place of digitization, security planning issues, etc.

2. Digital recording, which is the main digitization process according to the plan from phase 1 .

3. Data processing, which involves the modeling of the digitized object through the unification of partial scans, geometric data processing, texture data processing, texture mapping, etc.

\section{Three-dimensional (3D) digitization}

3D digitization of cultural content can be mainly categorized by the size of objects it is applied to. Due to technical limitations and application requirements, there must be a distinction between the digitization of objects and the digitization of monuments. Digitization of monuments is, in many cases, based on methods that involve traditional topographic techniques (due to the scale in this problem). On the other hand digitization of objects is a field of continuous research and development that can offer with many possibilities, again under the scope of a specific digitization plan. In the following paragraphs we briefly review some of these methods.

Table 1

The Nine-Criteria-Table for choosing an appropriate digitization system

\begin{tabular}{ll}
\hline No. & Criterion \\
\hline 1 & Cost \\
2 & Material of digitization subject \\
3 & Size of digitization subject \\
4 & Portability of equipment \\
5 & Accuracy of the system \\
6 & Texture acquisition \\
7 & Productivity of the technique \\
8 & Skill requirements \\
9 & Compliance of produced data with standards
\end{tabular}

\subsection{Digitization of objects}

\subsubsection{Laser scanning techniques}

Laser scanning techniques are based on a system with a laser source and an optical detector. The laser source emits light in the form of a line or a pattern on the surface of the objects and the optical detector (usually a digital camera) detects this line or pattern on the objects (Fig. 2). By applying the wellknown triangulation principle the system is able to extract the geometry of the objects. The advantage of using laser sources is that laser light is very bright and highly focused for long distances. As a result the emitted pattern can be always focused on the surface of the objects.

One of the most significant advantages of laser scanners is their high accuracy in geometry measurements. On the other hand, it should be noted that in many such systems, geometry can be extracted without any texture information. Additionally, special attention should be paid for surfaces with specific properties, such as reflectance and transparency. One other important aspect is the high cost of such devices, which renders this method useful to specific applications. Finally, the productivity of the method, as well as the portability, depends upon the used system and can vary significantly [1-3].

\subsubsection{Shape from structured light}

This method is based on projecting a specific pattern on the surface of the objects and trying to extract geometry information from the deformations of this pattern (Fig. 3). This method is also based on triangulation but does not need to use specific laser sources. In many cases this method is confused with the laser scanning methods and there are commercial systems that can not be absolutely categorized to the one or the other method.

The method works by projecting a specific predefined light pattern that covers the whole (or part of the) surface of the objects. This scene is then captured by a typical digital image detector and processed in order to deduce the geometry from the deformations of the pattern in the digital image. These patterns can be simple multiple fringes of different colors or complex patterns with curves, either time or space coded. This method is accompanied by texture acquisition and can lead to very impressive results in terms of accuracy and

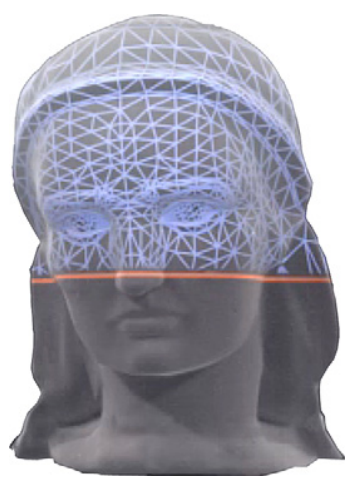

Fig. 2. Laser scanning of objects. 


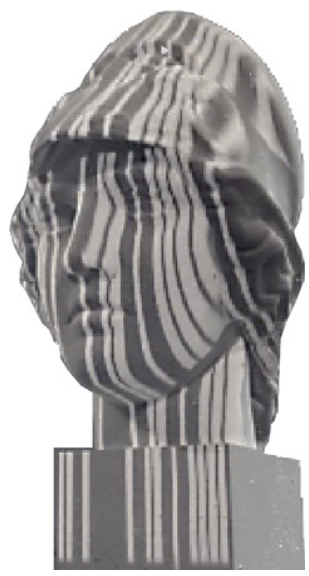

Fig. 3. Shape from structured light.

productivity. The systems are usually portable and easy to use. A lot of work is still being done to develop even more the resolution of the method, which is one of the main fields of research in 3D scanning today [4-16].

\subsubsection{Shape from silhouette}

This technique is based on multiple photographic capturing of the object from different viewing angles, and deducing the geometry from the object's silhouettes (Fig. 4). This is, actually, an old idea originating back to 1960 when Francois Villeme discovered a method called photo-sculpturing: 24 photographs covering the surface of the object are taken and are projected onto clay. This method regained interest about 100 years later with the advent of computers.

Recent improvements of this method use texture information to correct or enhance geometry with very interesting results in terms of the final recorded geometry. Shape from silhouette is an automated process with high productivity and relatively low cost. As of this moment it is very popular. It can capture both geometry and texture. It is portable and easy to use. The main disadvantage is the medium-to-low resolution in geometry measurements [17-23].

\subsubsection{Shape from stereo}

The main goal of this method is the extrapolation of as much geometry information as possible from only a pair of photographs taken from known angles and relative positions,

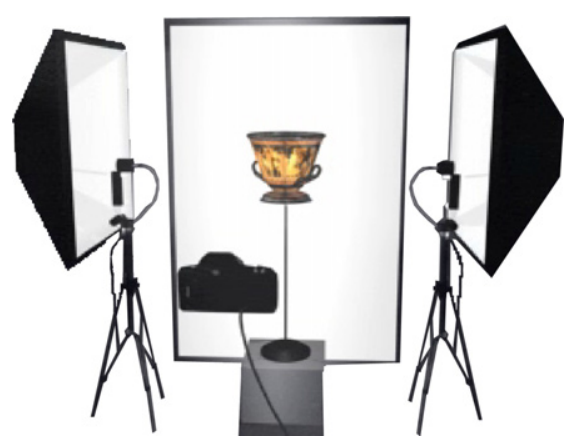

Fig. 4. Shape from silhouette. simulating the human visual system. Stereo-photography has a significant application in robotic and computer vision. It is based on taking pairs of photographs from slightly different angles. When certain parts of the object in the scene are visible to both photographs, specific algorithms from vision can be applied to extract the geometry of the object. The external as well as the internal parameters of the optical system are used for calibration. Calibration is critical in terms of achieving accurate measurements. The method can either be fully automated or manually operated. The final result is a depth map of the object in the scene, reflecting the distance of each recognized point on the surface of the object from the photographic sensor. Advantages of this method are the ability to capture both geometry and texture, the low cost and portability. A disadvantage of the method is its low resolution [24,25].

\subsubsection{Shape from video}

Shape from video is a variant of shape from stereo. Here the two photo cameras are replaced by a video camera that captures the object in a sequence of images from different views. A basic requirement for the application of this method is that the object is at complete rest and with no movable parts. The algorithms that are being used are similar to the ones in shape from stereo and are sensitive to noise in the video sequence. A key point in the process is the identification of common points between different images and the registration of these points onto a virtual 3D scene. The results are sometimes ambiguous due to the fact that there is no prior knowledge about the position of the camera or the objects. Advantages of this method can be considered the low cost, its portability and the ability to capture both texture and geometry. A significant disadvantage is the low resolution in capturing the geometry $[26,27]$.

\subsubsection{Shape from shading}

Shading plays an important role in depth perception. Many researchers have already tried to simulate the way the human visual system uses shading information to perceive the depth. This method requires the capturing of the object from one viewing angle. What should vary is the position of the light source, which would cause the shading to vary on the surface of the object. In this way, special algorithms could deduce the geometry of the surface of the object by using multiple photos of different shading conditions. The method is simple and has low cost. It captures both geometry and texture, with a minor disadvantage in capturing texture in shaded areas. It is portable but has the disadvantage of low accuracy. There are suggestions of using this method combined with other methods (such as shape from stereo) in order to enhance its accuracy [28].

\subsubsection{Shape from texture}

Texture can be a very significant source of information for the surface geometry. The calculation of 3D primitive shapes on a surface can be done if some prior knowledge exists about the surface texture. It is well known that the human visual 
system can easily identify surface geometry when the surface texture is homogeneous. Researchers tried to exploit this observation in order to simulate the process. Thus, the idea is to identify small structuring texture elements (texels) and to find their possible transformations in order to reproduce the whole surface of an object. These identified transformations are then used to extract the actual 3D surface geometry. The method is again photographic, simple and of low cost, but has limited applications (such as capture of fabric or human skin). It is portable and easy to use. On the other hand it has low accuracy [29].

\subsubsection{Shape from photometry}

Shape from photometry is a variant of shape from shading. Here the photos show the object from one viewing angle but in varying lighting conditions. Additionally, the usage of reference objects (or, in some cases, reference lighting sources) in the scene is critical, since they are used as calibration objects. Calibrated lights can improve significantly the result of the method but can only be found in special laboratories, so in this case the method is not portable. In studies against laser techniques there have been reports that favor this method in terms of the produced data volume and the immunity to laser limitations. Generally it can be regarded as portable, and it is easy to use and of low cost. The main disadvantage is its current need for a laboratory environment [30-32].

\subsubsection{Shape from focus}

Lately, a new possibility has attracted the interest of researchers: the possibility of exploiting the depth of field in a photo in order to deduce the 3D geometry of the scene. The method is recursive and is based on taking photos of an object while continuously adjusting the focus plane. By knowing the position of this focus plane (from the whole setup and the positioning of the system) we are able to map the focused pixels in an image on the correct position in the 3D depth map. The system, recursively, rebuilds the whole object geometry photo by photo. Resolution as well as accuracy is limited, but the results are, in general, "reliable". One limitation comes from the fact that in order to take photos with so limited a depth of field one might need a very special lens and a major application is in the usage of microscopes. The cost is relatively high, but the method is simple and easy to apply $[33,34]$.

\subsubsection{Shape from shadow}

Shape from shadow rebuilds the 3D model of an object by exploiting the deformation of the shadow of a known object which is projected onto the surface of the subject of digitization, when the light is moving. Evidently, this is a simple variant of the shape from structured light technique. The main advantage of this method is its low cost and the limited demand for computing power. It can reconstruct geometry even in nonvisible parts on the object, under certain assumptions about the object (or any prior knowledge). For this method, one might even find open source code on the Internet. The method has low accuracy [35].

\subsubsection{Contact systems}

Very often we might find digitization systems that use lasers mounted on some sort of arm with a high degree of freedom. This arm can either be operated manually or automatically, and through its internal positioning system carries geometry information to the controlling software. These systems are generally called Coordinate Measuring Machines (CMMs). Apart from their combined usage with laser devices, they can be operated autonomously. A spike can be mounted on them and by maintaining continuous contact with the object to be measured, very accurate geometry information can be recorded. The method is of high accuracy but very slow. There is also the disadvantage of having to be in contact with the objects, which is sometimes inadmissible [36].

\subsection{Digitization of monuments}

\subsubsection{Empirical techniques}

During an empirical recording of monuments, measurements are taken (by hand) of distances between characteristic points on the surface of the monument. The definition of the coordinates is done on an arbitrary coordinate system on a planar surface of the monument. The method is simple and productive, portable and of low cost. On the other hand it is of low accuracy and demanding in terms of time of physical presence near the monument. It can be successfully applied when a monument has simple shape, or there is a need for recording a sectional plan or sections of interiors [37].

\subsubsection{Topographic techniques}

The topographic method implements a 3D orthogonal coordinate system by using complicated and high-accuracy measuring devices. Mainly, this method uses a Geodesic Station, a system for measuring angles and distances of characteristic points on the surface of the monument, which are further transformed to coordinates in reference to the initial orthogonal coordinate system. The main advantage of the method is its high accuracy and objectivity of the measurements. It is reliable and it is easy to process its results. A disadvantage is the need for long physical presence near the monument, but it is one of the only methods to be used under difficult conditions, such as complex shape and difficult access. It is referred to as ideal for producing high-accuracy models of scale 1:50 or smaller [37].

\subsubsection{Laser scanning techniques}

Laser scanners can actually be considered as advanced geodesic stations and can be used to measure topographic quantities. They can measure the direction of a fictional optical line joining the characteristic points on the surface of the monument to a reference point on the measuring device (Fig. 5). Additionally these scanners can estimate their distance from these points. By applying the known triangulation principle they produce Cartesian coordinates automatically. The main advantage is the high accuracy and productivity, as well as the large volume of measurement data produced. It is reliable and objective. On the other hand it is a method 


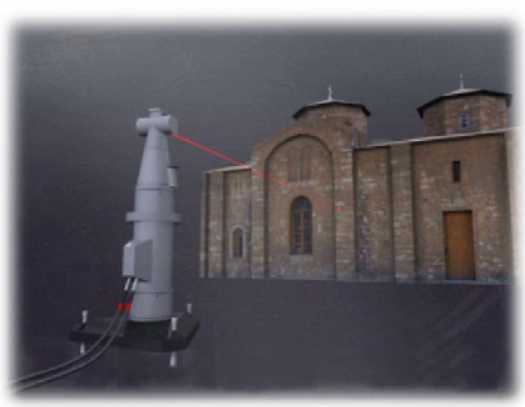

Fig. 5. Laser (range) scanning of monuments.

of high cost and difficulties in portability and autonomy. It can be applied on almost every monument digitization, but can experience interference from very bright light [38].

\subsubsection{Photogrammetry}

Common digital photos can be used, under suitable conditions, for measurements that can be of the accuracy obtained by the topographic methods. By applying orientation processes and transformations of digital photogrammetry it is possible to deduce $2 \mathrm{D}$ or $3 \mathrm{D}$ coordinates from one or two photos. The method is objective and reliable and can be aided by CAD software. It is relatively simple and has low cost. On the other hand it has to be combined with topographical or empirical measurements, and the final outcome is a function of the time spent. It can be used for complex objects with high surface detail, but since it is based on photos, there is a need for adequate space. It is also useful when direct access or contact to the monument is prohibited. It can be used to record stages of the monument in time. When combined with accurate measurements it can produce models of high accuracy for scales of 1:100 and even higher [37,39,40].

\section{Conclusions}

Complete digital recording of Cultural Heritage is a multidimensional process. It depends highly on the nature of the subject of recording as well as the purpose of its recording. In this work we made an attempt to summarize most of the methods available today for three-dimensional digitization that can be applied to digital cultural heritage recording.

\section{References}

[1] B. Curless, M. Levoy, Better optical triangulation through spacetime analysis, Proceedings of Fifth International Conference on Computer Vision 20-23 (June 1995) 987-994.

[2] M. Rioux, 1994. Digital 3-D imaging: theory and applications, in SPIE Proceedings, Videometrics III, International Symposium on Photonic and Sensors and Controls for Commercial Applications, Boston, 2650, pp. $2-15$.

[3] J.A. Beraldin, F. Blais, L. Cournoyer, G. Godin, M. Rioux, Active 3D sensing, SCUOLA NORMALE SUPERIORE PISA, Centro di Ricerche Informatiche per i Beni Culturali, 2000.

[4] J. Salvi, J. Pages, J. Batlle, Pattern codification strategies in structured light systems, Pattern Recognition 37 (4) (April 2004) 827-849.
[5] C. Rocchini, P. Cignoni, C. Montani, P. Pingi, R. Scopigno, A low cost 3D scanner based on structured light, in: A. Chalmers, T.-M. Rhyne (Eds.), EG 2001 Proceedings, vol. 20 (3), Blackwell Publishing, 2001, pp. 299-308.

[6] D. Caspi, N. Kiryati, J. Shamir, Range imaging with adaptive color structured light, Pattern analysis and machine intelligence 20 (5) (1998) 470-480.

[7] J. Gühring, Dense 3-d surface acquisition by structured light using offthe-shelf components, Videometrics and Optical Methods for 3D Shape Measurement 4309 (2001) 220-231.

[8] O. Hall-Holt, S. Rusinkiewicz, Stripe boundary codes for real-time structured-light range scanning of moving objects, in: The 8th IEEE International Conference on Computer Vision, 2001, pp. II: 359-366.

[9] N.G. Durdle, J. Thayyoor, V.J. Raso, An improved structured light technique for surface reconstruction of the human trunk, In: IEEE Canadian Conference on Electrical and Computer Engineering, 2 (1998) 874877.

[10] J. Salvi, J. Batlle, E. Mouaddib, A robust-coded pattern projection for dynamic 3d scene measurement, International Journal of Pattern Recognition Letters 19 (1998) 1055-1065.

[11] E.M. Petriu, Z. Sakr, H.J.W. Spoelder, A. Moica, Object recognition using pseudo-random color encoded structured light, in: Proceedings of the 17th IEEE Instrumentation and Measurement Technology Conference, vol. 3, 2000, pp. 1237-1241.

[12] L. Zhang, B. Curless, S.M. Seitz, Rapid shape acquisition using color structured light and multi-pass dynamic programming, International Symposium on 3D Data Processing Visualization and Transmission, Padova, Italy, 2002

[13] R.A. Morano, C. Ozturk, R. Conn, S. Dubin, S. Zietz, J. Nissanov, Structured light using pseudorandom codes, Pattern Analysis and Machine Intelligence 20 (3) (1998) 322-327.

[14] C. Wust, D.W. Capson, Surface profile measurement using color fringe projection, Machine Vision and Applications 4 (1991) 193-203.

[15] E. Horn, N. Kiryati, Toward optimal structured light patterns, Image and Vision Computing 17 (2) (1999) 87-97.

[16] J. Salvi, J. Pagès, J. Batlle, Pattern codification strategies in structured light systems, Pattern Recognition 37 (4) (2004) 827-849.

[17] S. Tosovic, R. Sablatnig, M. Kampel, On combining shape from silhouette and shape from structured light, in: H. Wildenauer, W. Kropatsch, (Eds.), Proc. of 7th Computer Vision Winter Workshop, 2002, pp. $108-118$

[18] A. Laurentini, The visual hull concept for silhouette-based image understanding, IEEE Transactions on Pattern Analysis and Machine Intelligence 16 (2) (1994) 150-162.

[19] A. Baumberg, A. Lyons, R. Taylor, 3D S.O.M.-A commercial software solution to 3D scanning, Vision, Video, and Graphics (2003), The Eurographics Association 2003. Eurographics Partner Event Vision, Video, and Graphics 2003. Bath UK.

[20] M. Potmesil, Generating octree models of 3D objects from their silhouettes in a sequence of images, CVGIP 40 (1987) 1-29.

[21] H. Noborio, S. Fukuda, S. Arimoto, Construction of the octree approximating three-dimensional objects by using multiple views, IEEE Trans, on PAMI 10 (1988) 769-782.

[22] N. Ahuja, J. Veenstra, Generating octrees from object silhouettes in orthographic views, IEEE Trans, Pattern Analysis and Machine Intelligence (1989) 137-149

[23] H.P.A. Lensch, W. Wolfgang Heidrich, H.-P. Seidel, A silhouette-Based algorithm for texture registration and stitching, Graphical Models 63 (4) (2001) 245-262.

[24] D. Scharstein, R. Szeliski, A Taxonomy and Evaluation of Dense Two-Frame Stereo Correspondence Algorithms, IJCV 2002.

[25] M. Bertozzi, A. Broggi, G. Conte, A. Fascioli, Stereo-Vision System performance analysis, Enabling Technologies for the PRASSI Autonomous Robot, ENEA, Rome, Italy, January 2002, pp. 68-73, ISBN: 8882860248.

[26] A. Chiuso, H. Jin, P. Favaro, S. Soatto, MFm: 3-D Motion and Structure from 2-D Motion Causally Integrated Over Time: Implementation, in: D. Vernon (Ed.), Lect. Notes in Computer Science 1843, Computer Vision-ECCV, 2000, pp. 734-750. 
[27] L. Van Gool, F. Defoort, M. Pollefeys, R. Koch, M. Proesmans, M. Vergauwen, Special Lecture: 3D Modeling for Communications, Computer Graphics International, Hannover, Germany, 22-26 June 1998, p. 482.

[28] R. Zhang, P.-S. Tsai, J.E. Cryer, M. Shah, Shape from shading: a survey, IEEE Transactions on Pattern Analysis and Machine Intelligence 21 (8) (1999).

[29] D.A. Forsyth, Shape from texture without boundaries, Proceedings of the 7th European Conference on Computer Vision Part III, 2002, pp. 225-239, ISBN: 3-540-43746-0.

[30] C.-Y. Chen, R. Klette, C.-F. Chen, 3D Reconstruction Using Shape from Photometric Stereo and Contours, Image and Vision Computing, Palmerston North (November 2003).

[31] R. Basri, D. Jacobs, Photometric Stereo with General, Unknown Lighting, IEEE Conference on Computer Vision and Pattern Recognition (2001).

[32] A. Hertzmann, S.M. Seitz, Shape and materials by example: a photometric stereo approach, Proceedings of CVPR 2003, IEEE Computer Society Conference on Computer Vision and Pattern Recognition.

[33] Y.Y. Schechner, Depth from defocus vs. stereo: how different really are they? International Journal of Computer Vision 89 (2000) 141-162.

[34] P. Favaro, Shape from Focus/Defocus, Washington University, Department of Electrical Engineering, 2002. http://homepages.inf.ed.ac.uk/rbf/ CVonline/LOCAL_COPIES/FAVARO1/dfdtutorial.html.
[35] J.-Y. Bouguet, P. Perona, 3D Photography on your desk, in Proceedings of the International Conference on Computer Vision, Bombay, India, January 1998.

[36] M. Nashman, T. Hong, W. Rippey, M. Herman, An integrated vision touch-probe system for dimensional inspection tasks, in Proceedings of the SME Applied Machine Vision '96 Conference, Cincinnati, OH, June 3-6, 1996.

[37] E. Livieratos, Empiric, topographic or photogrammetric recording? Answers to properly phrased questions, in Proceedings of the congress, "Terrestrial Photogrammetry and Geographic Information Systems for the documentation of the National Cultural Heritage", Thessaloniki, 1992.

[38] W. Boehler, A. Marbs, 3D scanning instruments, in Proceedings of the CIPA WG 6 International Workshop, "On Scanning For Cultural Heritage Recording", Corfu, 2002.

[39] K. Hanke, P. Grusenmeyer, Architectural Photogrammetry: Basic theory, Procedures, Tools, Tutorial of Architectural Photogrammetry, Corfu (September 2002).

[40] V. Tsioukas, P. Patias, Low cost 3D visualization and measuring 'tool' in the service of archaeological excavations, in Computer Applications and Quantitative Methods in Archaeology Conference, "The Digital Heritage of Archaeology”, Heraklion, 2002. 